\title{
Autologous hematopoietic stem cell transplant for progressive diffuse systemic sclerosis: procedural success and clinical outcome in 5-year follow-up
}

\author{
Katarzyna Pawlak-Buś s, $^{1,2}$ Wiktor Schmidt ${ }^{3}$, Michał Olejarz ${ }^{3}$, Anna Czyż ${ }^{4}$, Mieczysław Komarnicki ${ }^{4}$, \\ Piotr Leszczyński ${ }^{1,2}$ \\ ${ }^{1}$ Department of Rheumatology and Rehabilitation, Poznań University of Medical Sciences, Poland \\ ${ }^{2}$ Department of Rheumatology and Osteoporosis, J. Struś Memorial Multidisciplinary Municipal Hospital in Poznań, Poland \\ ${ }^{3}$ Student Department of Rheumatology, Metabolic Bone and Connective Tissue Disorders, Poznań University of Medical Sciences, Poland \\ ${ }^{4}$ Department of Haematology and Stem Cell Transplantation, Poznań University of Medical Sciences, Poland
}

\begin{abstract}
Systemic sclerosis is an autoimmune connective tissue disease affecting both skin and internal organs. Progressive disease with multiple organ involvement is considered to have a poor prognosis. Treatment possibilities are limited, but certain patients may benefit from autologous hematopoietic stem cell transplantation (auto-HSCT).

We report a case of a 30-year-old woman with progressive diffuse systemic sclerosis treated with parenteral cyclophosphamide with unsatisfactory results. Due to progression of the disease and lack of alternative therapies auto-HSCT was performed.

After instituting treatment with autologous hematopoietic stem cell transplantation no immunosuppressive therapy has been required during 5-year follow-up. Improvement in exertion tolerance, partial regression of skin lesions and stabilization of pulmonary and cardiovascular changes were observed. Currently therapeutic options in patients with progressive systemic sclerosis are limited. Hematopoietic stem cell transplantation might become an alternative therapeutic solution not only in the early phase of the disease but also among selected patients with progressive systemic sclerosis resistant to standard therapy.
\end{abstract}

Key words: scleroderma, progressive systemic sclerosis, stem cell transplantation, hematopoietic stem cells.

\section{Introduction}

Systemic sclerosis (SSc) is an autoimmune connective tissue disease characterized by dermal and internal symptoms and a characteristic antibody profile (anticentromere - ACA, antitopoisomerase I - ATA, Scl-70, anti-RNA polymerase III - ARA). Its pathophysiology comprises vasculopathy, fibrosis and immune dysregulation. The initial vascular insult activates endothelium and promotes endothelial-mesenchymal transition, a process of transdifferentiation to mesenchymal cells such as fibroblasts and myofibroblasts. Dysregulation of the transforming growth factor $\beta$ (TGF- $\beta$ ) pathway and imbalance of vasodilators and vasoconstrictors bring about obliterative vasculopathy with interstitial and vascular fibrosis leading to luminal stenosis. This results in tissue ischemia and decrease of the blood vessel count. Predominant Th2 cytokine profile, alternatively activated macrophages and altered B cell function with their chronic activation and autoantibody production are major immune dysfunctions promoting fibrosis [1, 2].

The most typical symptoms of SSc emerge from dermal and vascular lesions. The Raynaud phenome- 
non occurs in the majority of cases and may precede development of other features. Skin thickening can affect the whole body; however, the most specific region for diffuse cutaneous SSc (dcSSc) is the proximal part of the limbs. The disease may also involve internal organs, predominantly the lungs, heart, kidneys or gastrointestinal tract. Diagnosis of systemic sclerosis can be supported by American College of Rheumatology/European League Against Rheumatism (ACR/EULAR) 2013 classification criteria [3]. Criteria developed by LeRoy et al. are useful in diagnosing the disease in its early stages [4]. Disease activity can be measured with the revised European Scleroderma Trials and Research group (EUSTAR) 2017 Disease Activity Index, with a score of 2.5 or greater indicating active disease [5].

The mortality in dcSSc is still high, with 85.5\% 5-year, 71.6\% 10-year and 55.1\% 15-year survival rates [6]. The most common SSc-related causes of death are pulmonary fibrosis (19\%), pulmonary arterial hypertension (14\%) and cardiac events (6\%). Scleroderma renal crisis which contributed significantly to mortality among the patients with SSc in the past is currently a rarity [7].

The standard treatment for patients at high risk of organ failure and death, especially with lung involvement, is cyclophosphamide (CTX), although the response varies greatly and is often insufficient. The first transplantations in autoimmune disorders were performed in 1994 [8]. The procedure was considered experimental for a very long time until the results of two recent randomized controlled trials (RCTs) proving its efficacy in SSc emerged $[9,10]$.

So far until the year 2018 several autologous hematopoietic stem cell transplantations (auto-HSCTs) for SSc have been performed in Poland and the transplantation carried out in Poznań was the sole published procedure using anti-thymocyte globulin (ATG) in the conditioning regimen.

\section{Case report}

A previously healthy 30-year-old Caucasian woman was diagnosed with systemic sclerosis in 2004 due to the occurrence of chronic Raynaud phenomenon (from 2002), typical skin lesions and a characteristic but heterogeneous antibody profile with positive $\mathrm{Scl}-70$, centromere B, Ro-52, nucleosome and AMA-M2 autoantibodies. Early diagnosis of dcSSc was supported by criteria of LeRoy et al. [4]. Apart from positive antinuclear antibodies there were no disturbances in laboratory results, with inflammatory indices remaining within the normal range (ESR $10 \mathrm{~mm} / \mathrm{h}$, CRP $0.2 \mathrm{mg} / \mathrm{dl}$ ).

Intensive and many-years-long intravenous treatment with 800-1000 mg CTX monthly was instituted. Despite treatment, progression occurred and organ involvement extended. Skin symptoms included fibrosis, telangiectasias, finger shortening with onycholysis and difficult to treat digital ulcers. Organ symptoms comprised interstitial lung disease (ground-glass in highresolution computed tomography - HRCT and $55.7 \%$ of predicted carbon monoxide diffusion capacity - DLCO), increased right ventricular systolic pressure (RVSP - $40 \mathrm{~mm} \mathrm{Hg}$ ) and heart palpitations. Left ventricular ejection fraction (LVEF) remained within the normal range $-63 \%$. Dysphagia and postprandial vomiting were symptoms that negatively influenced the patient's quality of life.

Due to progression despite treatment, poor prognosis and lack of alternative therapies the patient was qualified to be treated with auto-HSCT as a salvage therapy in 2011. After having acquired informed consent, screening for exclusion criteria for HSCT was performed including DLCO, heart ultrasonography, electrocardiography and creatinine clearance. The patient was evaluated as eligible for HSCT, as dysfunction of organs did not exceed the values in exclusion criteria of the American Scleroderma Stem Cell versus Immune Suppression Trial (ASSIST) and the Autologous Stem Cell Transplantation International Scleroderma Trial (ASTIS) trials and inclusion criteria were fulfilled except for duration of the disease (7 years from first non-Raynaud symptom - puffy fingers in January 2004) and a cumulative dose of CTX $(29400 \mathrm{mg})[9,10]$.

Hematopoietic stem cell mobilization was achieved with $4 \mathrm{~g} / \mathrm{m}^{2} \mathrm{CTX}$ and subsequent infusion of granulocyte colony-stimulating factor (G-CSF). High-dose chemotherapy with $200 \mathrm{mg} / \mathrm{kg}$ CTX and $60 \mathrm{mg} / \mathrm{kg}$ antithymocyte globulin (ATG) was instituted afterwards as conditioning. Despite uroprotection with mesna and hyperhydration, the patient suffered from hemorrhagic cystitis that was treated with bladder irrigation with success. Due to this adverse effect the doses had to be downgraded to $150 \mathrm{mg} / \mathrm{kg}$ CTX and $40 \mathrm{mg} / \mathrm{kg}$ ATG. The transplantation procedure was carried out on 17 January 2011. Granulopoiesis reconstitution was achieved 9 days after HSCT. The procedure ended successfully.

Five-year follow-up provides satisfactory effects of auto-HSCT as shown in Table I.

Skin fibrosis assessed with modified Rodnan skin score was downgraded (mRSS 15 vs. 31). Improvement of skin lesions was noted mainly on the trunk and proximal parts of the limbs. Facial skin changes and fibrosis of distal parts of upper limbs stabilized. No new finger ulcerations developed after HSCT was performed.

Pulmonary function tests proved stabilization of interstitial lung disease (DLCO 55.3\% vs. 55.7\% predicted), and forced vital capacity (FVC) and forced expiratory volume in 1 second (FEV1) remained in the normal range 
Table I. Major parameters evaluated in 5-year follow-up after autologous hematopoietic stem cell transplantation

\begin{tabular}{|lcc|}
\hline Parameter & 2011 & 2016 \\
\hline Skin fibrosis (mRSS) & 31 & 16 \\
\hline DLCO & $55.7 \%$ predicted & $55.3 \%$ predicted \\
\hline RVSP & $40 \mathrm{~mm} \mathrm{Hg}$ & $28 \mathrm{~mm} \mathrm{Hg}$ \\
\hline LVEF & $63 \%$ & $60 \%$ \\
\hline $\begin{array}{l}\text { EUSTAR 2017 Disease } \\
\text { Activity Index (assessed } \\
\text { retrospectively) }\end{array}$ & 5.5 & 2.34 \\
\hline
\end{tabular}

DLCO - carbon monoxide diffusion capacity; RVSP - right ventricular systolic pressure; LVEF - left ventricular ejection fraction.

just as before the transplant. HRCT scans showed no progression of interstitial lung changes. RVSP decreased from 40 to $28 \mathrm{~mm} \mathrm{Hg}$. There was no significant deterioration in left ventricle function (LVEF 60 vs. 63\%).

Improvement in exertion tolerance and exertional dyspnea was noted. Symptoms from the gastrointestinal tract such as dysphagia and postprandial vomiting have improved, but pathologic changes in the esophagus have remained. Antinuclear antibodies have been undetectable through the whole follow-up, while ESR and CRP have remained in the normal range $(13 \mathrm{~mm} / \mathrm{h}$ and $0.1 \mathrm{mg} / \mathrm{dl}$ respectively). As an adverse effect of highdose chemotherapy the patient suffered from amenorrhea. Disease activity measured retrospectively with the revised EUSTAR 2017 Disease Activity Index significantly decreased from 5.5 to 2.34 and now can be stated as stable and inactive [5].

No immunosuppressive medication has been required up to now; thus monthly hospitalizations have been consigned to history. The patient stated that possibility to avoid frequent and long hospital visits and no requirement for immunosuppressive agents were the most important factors which improved the quality of her life. This 5-year follow-up after HSCT of our patient with dcSSc proves it was a medical success.

\section{Discussion}

No RCT results in the field of HSCT in progressive dcSSc had been published by the time the transplant was performed in our patient. However, data collected in six multicenter phase I/II studies provided encouraging findings which formed the basis for prospective RCTs that were launched in 2000s [11]. Two of them have been completed and published: ASSIST - an open-label, phase II RCT on 55 patients, and ASTIS - a phase III, multicenter, open-label RCT on 156 patients $[9,10]$. Both studies currently provide the best available evidence for the efficacy and safety of auto-HSCT with a non-irradiation conditioning regimen in $\mathrm{dcSSC}$.

Autologous HSCT in dcSSc in the majority of cases is performed using peripheral blood stem cells rather than blood marrow. Mobilization is obtained with CTX $2-4 \mathrm{~g} / \mathrm{m}^{2}$ followed by G-CSF 5-10 $\mathrm{\mu g} / \mathrm{kg}$. The recommended conditioning regimen should be of intermediate intensity; most frequently it consists of CTX $200 \mathrm{mg} / \mathrm{kg}$ with polyclonal or monoclonal anti-T-cell serotherapy. Both alemtuzumab and ATG may be considered, depending on availability; however, in our case ATG was chosen as in ongoing trials in those days for the better outcome $[9,10,12]$.

The HSCT procedure is associated with a high treatmentrelated mortality (TRM), which ranges from $6 \%$ to $17 \%$ for non-irradiation based regimens [13]. Causes of TRM in HSCT include Epstein-Barr virus reactivation, lymphoma, heart failure, myocardial infarction and acute respiratory distress syndrome (ARDS) [10]. Lowering of TRM can be achieved via extended cardiopulmonary work-up including heart MRI and catheterization with fluid challenge, as most of the patients who died after this procedure did so due to poor cardiac conditions before transplant, as was proven in a retrospective analysis of 90 patients after HSCT by Burt et al. [14]. Thus, comprehensive extended screening in selection of patients at risk of organ failure yet without severe organ dysfunction, who would benefit ultimately from such treatment, is essential and highly recommended nowadays $[12,15]$.

Our patient fulfilled the inclusion criteria of ASSIST and ASTIS trials except for disease duration (counted from the first non-Raynaud symptoms) longer than 5 years and cumulative dose of CTX higher than $5 \mathrm{~g}[9,10]$. Therapy with CTX had been well tolerated up to then but brought unsatisfactory results and did not cease or delay organ damage. Progression was significant and prognosis was very poor; thus HSCT was considered as salvage therapy with no comparable alternatives. Comprehensive examination in accordance with European Society for Blood and Marrow Transplantation (EBMT) guidelines proved that organ damage had not yet been too severe to fulfill any of organ-function related exclusion criteria of abovementioned trials; thus the patient was stated to be eligible for the transplant $[9,10]$. In our opinion neither very long disease duration nor very high cumulative CTX dose is an absolute contraindication to auto-HSCT in real life, and this distinguishes our case from clinical trials.

The ASSIST trial showed that auto-HSCT was superior to CTX (1000 mg/m²/month for 6 months) in skin score and lung function improvement (FVC, diseased-lung volume) [9]. The ASTIS trial in turn provided evidence that auto-HSCT was superior to CTX $(750 \mathrm{mg} /$ 
$\mathrm{m}^{2} /$ month for 12 months) in the long term (median follow-up 5.8 years) event-free survival 22 vs. 31 with adverse events described as death or major organ failure. However, the event occurrence in the first year after transplant was higher in the HSCT group due to TRM associated with the procedure (13 vs. 8). The HSCT was also proved superior to CTX in improvement of skin fibrosis (mRSS 19.9 in the HSCT group vs. 8.8 in the control group), functional ability, quality of life, and lung function (FVC and total lung capacity - TLC, though no significant effect on DLCO was found) [10]. In the retrospective analysis by Burt et al. relapse-free survival was $70 \%$ at 5 years. Mean mRSS was downgraded by 15.2 points (24.1 vs. 8.9) 5 years after HSCT. Among lung function markers, FVC improvement after HSCT was significant up to 3 years, but not after 5 years. TLC and DLCO did not change significantly for the entire group. Significant DLCO enhancement was observed only among patients with normal baseline electrocardiograph or echocardiogram [14]. The results of the abovementioned studies correspond with the clinical findings in our patient, whose event- and relapse-free survival is already over 5 years long. The mRSS has decreased from 31 to 16, lung function has not changed significantly either functionally (DLCO, FVC) or radiologically (HRCT) and quality of life has been improved, mainly due to better exertion tolerance and lessening of dysphagia and postprandial symptoms.

Studies modifying protocols from ASSIST and ASTIS - Autologous Stem Cell Systemic Sclerosis Immune Suppression Trial (ASSIST IIb), Autologous Stem Cell Transplantation for Progressive Systemic Sclerosis (AST-MOMA) and Scleroderma Treatment With Autologous Transplant (STAT) in order to achieve overall survival improvement with less toxicity are currently under investigation. Disease duration in AST-MOMA inclusion criteria must be less than 7 years, while in ASSIST IIb and STAT it is not listed at all [16-18]. The cumulative dose of CTX is not included in exclusion criteria of ASSIST IIb and STAT studies, while in the AST-MOMA trial a cumulative dose of > $50 \mathrm{~g}$ CTX is listed as a relative exclusion criterion [16-18]. Inclusion and exclusion criteria in ongoing RCTs tend to be more favorable to patients with similar clinical features as presented by our patient. Patients with a longer history of SSc and larger cumulative CTX doses are currently being included in RCTs and HSCT may become an important alternative treatment not only in early disease, but also in progressive disease that lasts longer and does not respond well to standard therapy.

The first EBMT recommendations on HSCT in severe autoimmune diseases were published in 1997, and the latest update came out in 2012 [8, 12]. Patients with dcSSc defined as eligible to be treated with this meth- od are those with disease duration equal to or less than 5 years since the first non-Raynaud symptoms with an mRSS above 14 plus major organ involvement - pulmonary, cardiac or renal without severe organ dysfunction or infections listed in the exclusion criteria [12]. Respectively evaluated, our patient fulfilled the inclusion criteria except for disease duration less than 5 years and the organ damage was not too severe to match any of the exclusion criteria. When more data become available, the disease duration excluding a patient from such treatment may be revised and prolonged in updated guidelines.

In 2017 new recommendations on cardiopulmonary assessment of patients with SSC for HSCT were published by the EBMT ADWP Scleroderma Working Group. The pre-transplant assessment was broadened compared with assessment in our patient that was standard in 2011. Trans-thoracic echocardiography (TTE) with Doppler and tissue Doppler plus dobutamine stress test, cardiac magnetic resonance with contrast (CMR), cardiac catheterization with fluid challenge, HRCT, ECG and pulmonary function testing (including DLCO, spirometry and body plethysmography) should be performed in order to decrease the risk of transplant-related toxicity and morbidity [19].

In 2016 HSCT was added to EULAR recommendations for the treatment of patients with rapidly progressive SSc at risk of organ failure after comprehensive screening in order to minimize the risk of early TRM [15]. We still lack data about efficacy and safety of HSCT in patients with progressive disease resistant to standard therapy with disease duration longer than 5 years at risk of organ failure yet without it. This may contribute to minimizing the toxicity and maximizing the benefit from HSCT in the era of a growing requirement for effective alternative therapies such as transplantations performed among patients like ours. It seems a promising option in cases such as the present one, inasmuch as no comparable alternatives are currently available. Biologic or other new disease-modifying drugs are not as widely or effectively used in SSc, unlike in other rheumatic diseases such as rheumatoid arthritis. Organ-specific therapy remains standard, but HSCT is the only method that can reverse and stabilize the disease for a long time.

\section{Conclusions}

Autologous HSCT seems to be currently the most effective treatment of progressive dcSSc. It can reverse or at least stabilize SSc symptoms for a long time. It was included in the 2016 update of the EULAR recommendations for the treatment of rapidly progressive systemic sclerosis with internal organ involvement [15]. 
Comparison with intravenous cyclophosphamide pulses suggests that auto-HSCT induces more robust and sustained responses of skin involvement and functional status. The benefit-to-risk ratio needs to be assessed accurately in each case, due to a high level of toxicity and great risk of treatment-related mortality, which hopefully appears to decrease with growing experience. As some big randomized controlled trials are still ongoing or awaiting publication, with each year our knowledge about this method rises and we have more data and evidence optimizing its use. The HSCT seems effective not only among patients with early rapidly progressive disease but also with progressive disease resistant to standard therapy, such as in the present case; thus indications for instituting such treatment might be broadened in the near future.

\section{The authors declare no conflict of interest.}

\section{References}

1. Abraham DJ, Eckes B, Rajkumar V, et al. New developments in fibroblast and myofibroblast biology: implications for fibrosis and scleroderma. Curr Rheumatol Rep 2007; 9: 136-143.

2. Katsumoto RT, Whitfield ML, Connolly MK. The Pathogenesis of Systemic Sclerosis. Annu Rev Pathol 2011; 6: 509-537.

3. van den Hoogen F, Khanna D, Fransen J, et al. 2013 Classification Criteria for Systemic Sclerosis: An American College of Rheumatology/European League Against Rheumatism Collaborative Initiative. Arthritis Rheum 2013; 65: 2737-2747.

4. LeRoy EC, Medsger TA. Criteria for the classification of early systemic sclerosis. J Rheumatol 2001; 28: 1573-1576.

5. Valentini G, Iudici M, Walker UA, et al. The European Scleroderma Trials and Research group (EUSTAR) task force for the development of revised activity criteria for systemic sclerosis: derivation and validation of a preliminarily revised EUSTAR activity index. Ann Rheum Dis 2017; 76: 270-276.

6. Nihtyanova SI, Schreiber BE, Ong VH, et al. Prediction of pulmonary complications and long-term survival in systemic sclerosis. Arthritis Rheumatol 2014; 66: 1625-1635.

7. Tyndall AJ, Bannert B, Vonk M, et al. Causes and risk factors for death in systemic sclerosis: a study from the EULAR Scleroderma Trials and Research (EUSTAR) database. Ann Rheum Dis 2010; 69: 1809-1815.

8. Tyndall A, Gratwohl A. Blood and marrow stem cell transplants in auto-immune disease: a consensus report written on behalf of the European League against Rheumatism (EULAR) and the European Group for Blood and Marrow Transplantation (EBMT). Bone Marrow Transplant 1997; 19: 643-645.

9. Burt RK, Shah SJ, Dill K, et al. Autologous non-myeloablative haemopoietic stem-cell transplantation compared with pulse cyclophosphamide once per month for systemic sclerosis (ASSIST): an open-label, randomised phase 2 trial. Lancet 2011; 378: 498-506.

10.van Laar JM, Farge D, Sont JK, et al. Autologous hematopoietic stem cell transplantation vs intravenous pulse cyclophospha- mide in diffuse cutaneous systemic sclerosis: a randomized clinical trial. JAMA 2014; 311: 2490-2498.

11. Burt RK, Milanetti F. Hematopoietic stem cell transplantation for systemic sclerosis: history and current status. Curr Opin Rheumatol 2011; 23: 519-529.

12. Snowden JA, Saccardi R, Allez M, et al. Haematopoietic SCT in severe autoimmune diseases: updated guidelines of the European Group for Blood and Marrow Transplantation. Bone Marrow Transplant 2012; 47: 770-790.

13.van Laar JM, Sullivan K. Stem cell transplantation in systemic sclerosis. Curr Opin Rheumatol 2013; 25: 719-725.

14. Burt RK, Oliveira MC, Shah SJ, et al. Cardiac involvement and treatment-related mortality after non-myeloablative haemopoietic stem-cell transplantation with unselected autologous peripheral blood for patients with systemic sclerosis: a retrospective analysis. Lancet 2013; 381: 1116-1124.

15. Kowal-Bielecka O, Fransen J, Avouac J, et al. Update of EULAR recommendations for the treatment of systemic sclerosis. Ann Rheum Dis 2017; 76: 1327-1339.

16. Northwestern University. Autologous Stem Cell Systemic Sclerosis Immune Suppression Trial (ASSIST IIb). U. S. National Library of Medicine, Bethesda 2000-2017. Available from: https://clinicaltrials.gov/ct2/show/NCT01445821 NLM Identifier: NCT01445821.

17. University Hospital Tuebingen. Autologous Stem Cell Transplantation for Progressive Systemic Sclerosis (AST-MOMA). U. S. National Library of Medicine, Bethesda 2000-2017. Available from: https://clinicaltrials.gov/ct2/show/NCT01895244 NLM Identifier: NCT01895244.

18. Fred Hutchinson Cancer Research Center. Scleroderma Treatment With Autologous Transplant (STAT) Study. U. S. National Library of Medicine, Bethesda 2000-2017. Available from: https://clinicaltrials.gov/ct2/show/NCT01314100 NLM Identifier: NCT01314100.

19. Farge D, Burt RK, Oliveira MC, et al. Cardiopulmonary assessment of patients with systemic sclerosis for hematopoietic stem cell transplantation: recommendations from the European Society for Blood and Marrow Transplantation Autoimmune Diseases Working Party and collaborating partners. Bone Marrow Transplant 2017; 52: 1495-1503. 